\title{
A Newly Developed Radio Frequency Wireless Passive Highly Sensitive Strain Transducer
}

\author{
Trang T. Thai, Herve Aubert, Patrick Pons, Robert Plana \\ CNRS-LAAS; Uni. of Toulouse; UPS, INSA, INP, ISAE; \\ F-31077 Toulouse Cedex 4, France \\ trang.thai@gatech.edu
}

\author{
Trang T. Thai, Manos. M. Tentzeris \\ School of ECE, Georgia Institute of Technology \\ Atlanta, GA 30332, U.S.A. \\ Gerald R. DeJean \\ Microsoft Research \\ One Microsoft Way, Redmond, WA 98052, U.S.A.
}

\begin{abstract}
A novel design of a highly sensitive wireless passive radio frequency strain transducer is presented based on a patch antenna loaded with an open loop that is capable of sensing strain remotely. The new idea of utilizing a cantilever at the gap of the open loop significantly improves the sensitivity of resonant frequency shifts. In measurements, the prototypes achieve a sensitivity of $1.26 \%$ frequency shift per $1 \%$ strain, while in simulations the new design achieves a theoretical sensitivity up to 4 times higher than existing designs of RF passive wireless strain transducers. The ground plane allows for the sensitivity of the sensor to be independent from the applied surface. An implementation example of the passive remote sensing system based on the proposed strain transducer is also discussed as a proof-of-concept.
\end{abstract}

\section{INTRODUCTION}

Strain sensing has been crucial in structural health monitoring especially in aerospace applications. The concept of Radio Frequency (RF) transducer has been introduced only recently and has been shown to enable many features on sensing such as wireless, passive, and miniaturized simultaneously, which was not feasible with conventional sensors. A few RF strain sensors have been proposed that detect the strain by resonant frequency shift of the resonating structures usually from the backscattered signals with parameter such as Radar Cross Section (RCS). However, these designs [1-3] yield rather low sensitivity in terms of frequency shift per strain percentage. In aerospace, strain is especially a sensitive parameter for operations of aircrafts, the conditions of wings and blades, as well as fatigue of the aircraft body. Strain sensors have been widely developed utilizing different techniques, among which the most common types are resistive and capacitive gages which typically require wiring, and/or complex circuits adding weight penalty that are especially highly undesirable for implementation on aircraft wings or helicopter blades as well as complicating maintenance. Although some wireless sensors have been reported [4-5], they usually include an embedded microprocessor and a radio frequency module to be integrated with the transducer which requires battery power to operate, thus unsuitable for long term monitoring due to limited lifetime and also more frequent maintenance and replacement. Other sensing techniques such as optical fiber-based are compact, lightweight, remote sensing capable, but expensive. Therefore, wireless passive strain sensors are highly desirable which can address those mentioned problems. The new design introduced here improve the sensitivity theoretically 4 times higher than existing designs because the new idea enables a new dimension of engineered sensitivity in this type of sensing. The sensitivity of the proposed design is independent from the material of the applied surface since it has a ground plane in design. As an example for a specific passive implementation, the sensor can be interrogated by radar to monitor its RCS level change as an indicator of strain.

\section{DESIGN AND PRINCIPLES OF OPERATION}

A new strain transducer design is introduced here based on a patch antenna loaded with an open loop and a cantilever (Fig. 1). The resonances of the patch depend on the impedance of the loaded loop determined by the loop length and the cantilever gap capacitance. The resonant frequency of the open loop, which is due to the length of the loop, is designed to be close to the dominant resonant frequency of the patch along $\mathrm{x}$ direction (Fig. 2). As a result, the structure generates a dual frequency response, in which the separation of the two frequencies is strongly influenced by the capacitance located on the open ring. That means the resonant peaks, especially the one responsible by the open loop, are highly sensitive to the capacitance value at the gap. The open loop here is modified from a traditional open loop, which is the key idea introduced in this work for sensitivity improvement.

Strain is measured by the deformation of a material volume along the strain direction. It is denoted as $\varepsilon \mathrm{L}=\Delta \mathrm{L} / \mathrm{L}$. 
The length Lo in the stretched direction is deformed into $\mathrm{L}$ as shown in Eq. 1. Strain is unit less and usually measured in \% or microstrain $(\mu \varepsilon)$ which is $10^{-3}$ of $\Delta \mathrm{L} / \mathrm{L}$. Therefore, any resonant or antenna structure attempting to transform this change directly into a detectable RF signal is inherently limited as observed in the existing designs [1-3]. To solve this problem, we utilize a cantilever implemented on the open loop that is loaded on a patch antenna instead of using the patch itself alone as in [1].

$$
L=\left(1+\varepsilon_{L}\right) L_{0}
$$

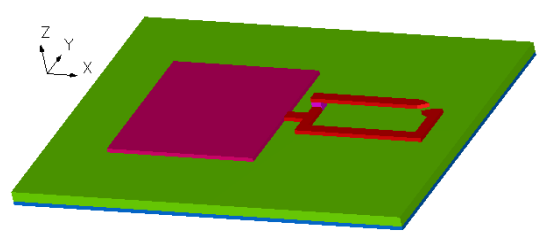

Fig. 1. 3D view of the Strain sensor.

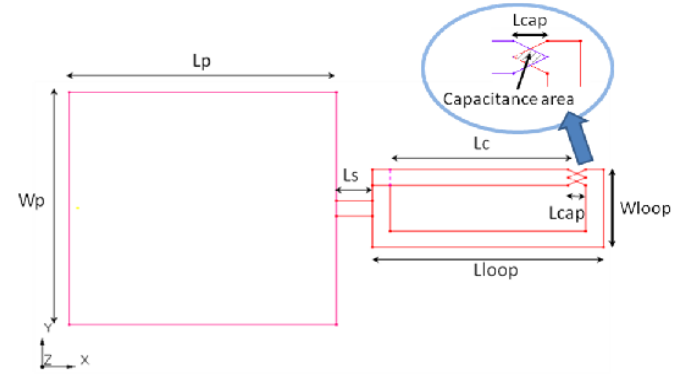

Fig. 2. Top view of the Strain sensor.

The essential idea in the sensitivity improvement is to have the cantilever shorted electrically and fixed mechanically at one end of the open loop, while the other end is free and separated electrically from the loop end to form a parallel plate capacitor. Therefore, as a certain $\mathrm{k} \%$ strain is induced on the substrate along $\mathrm{x}$ direction (Fig. 3), Lgap is increased by $\Delta$ Lgap $=\mathrm{k} \%$ Lgap. Because the cantilever is fixed to the surface only at one end, it is made free from this strain, thus its length, Lc, is constant. Therefore the entire length deformation, $\Delta$ Lgap is transferred to the change in Lcap (Fig. 3). Effectively, the capacitance is modified by ( $\mathrm{k} \%$ Lgap) instead of ( $\mathrm{k} \%$ Lcap), where Lgap can be engineered to be significantly larger than Lcap as shown in Fig. 2. The use of triangular tips at the loop gap is to further increase the rate of change of the capacitance with a given rate of change of Lcap.

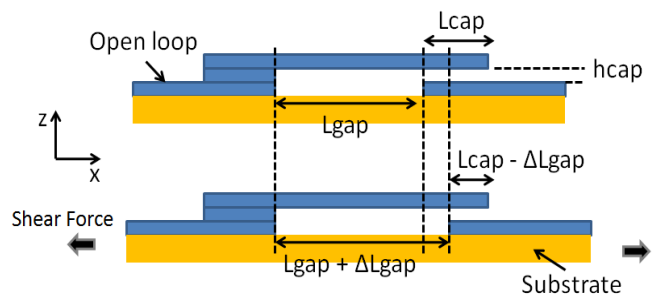

Fig. 3. Cross section view of the cantilever implemented on the modified loop loaded in the new strain sensor.

\section{Simulation SETUP AND RESUlts}

The effects of $1 \%$ strain are modeled in RF simulations by applying an increase of $1 \%$ in length is applied to each length along the elongation (direction of applied force) of the parameters Lp, Ls, and Lloop (Fig. 2). Since Lc is fixed and Lloop is increased by $1 \%$, Lcap is decreased by $1 \%$ of Lloop. Additionally, the substrate under strain also experiences the Poisson's effect, which essentially causes a contraction in the direction transverse to the strain, thus dimension Wpatch is effectively decreased. Poisson's effect is the ratio of this contraction to the extensions caused by strain. The Poisson's effect can be calculated as $\mathrm{w}=\left(1-\mathrm{v}_{\mathrm{p}} \varepsilon_{\mathrm{L}}\right) \mathrm{w}_{\mathrm{o}}$, in which $\mathrm{w}$ denotes the width of dimension that is transverse to the strain direction, vp is the Poisson's ratio of the Kapton, $\varepsilon \mathrm{L}$ is the applied strain. Other parameters including the Poisson's effect on the substrate thickness and the width of the open loop are negligible to the RF response of the structure, thus are not accounted for in simulations. Different models with different Lc values, are simulated. Note that, while other values are the same in the model for a given Lc including non-strain and under strain conditions, different Lc values effectively yield different Lcap values as illustrated in Fig. 4.

TABLE I

Summary of Simulation Parameters

\begin{tabular}{|c|c|c|}
\hline Dimensions (um) & No strain & Strain 1\% \\
\hline Lpatch (Fig. 2) & 26000 & 26260 \\
\hline Wpatch (Fig. 2) & 25000 & 24925 \\
\hline Ls (Fig. 2) & 2000 & 2020 \\
\hline Lloop (Fig. 2) & 13000 & 13130 \\
\hline Wloop (Fig. 2) & 5000 & 5000 \\
\hline c (width of stub and loop) & 1000 & 1000 \\
\hline hsub (substrate thickness) & 100 & 100 \\
\hline hcap (parallel plate capacitance gap) & 10 & 10 \\
\hline
\end{tabular}
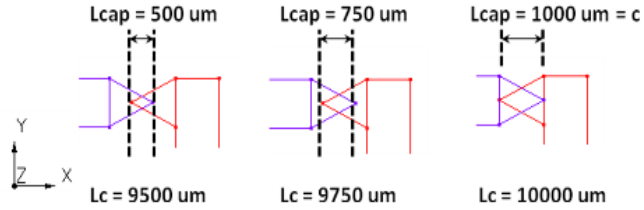

Fig. 4. Topview of the cantilever from top view in different strain conditions.

The magnitude of the reflection ratio $|\mathrm{S} 11|$ simulations results are presented in Fig. 5 for each Lc values of 500 um, $750 \mathrm{um}$, and $10000 \mathrm{um}$, with each Lc value, both strain free and $1 \%$ strain conditions are simulated using parameters given in Table I. The gap between the two parallel surfaces, the lower face of the cantilever free end and the upper face of the open loop end, is set to $10 \mathrm{um}$. This parameter is highly critical for the sensitivity of the strain transducer because it determines the initial gap capacitance. The transducer is designed to operate around $3 \mathrm{GHz}$, on Kapton substrate $100 \mathrm{um}$ thick with dielectric constant of 3.4. The simulations are performed in CST Microstripes software. The $|\mathrm{S} 11|$ results in Fig. 5 show that each model has a dual frequency response with two close resonant frequencies. The sensitivity of the transducer depends on the value of Lc, which determines the 
initial capacitance and rate of change of the capacitance as Lcap is decreased. Here, the highest sensitivity that can be achieved is found to be Lc of $9750 \mathrm{um}$, which induces a frequency shift of $125 \mathrm{MHz}$ at $2.72 \mathrm{GHz}$, or $4.6 \%$ frequency shift per $1 \%$ strain. This sensitivity is more than 4 times the sensitivity achieved by rectangular and circular patch antennas previously proposed, which rely only on the change in dimensions of the patches and achieved a sensitivity of only $0.9-1.0 \%$ frequency shift per $1 \%$ strain [1-3]. It is observed that as the strain is applied, the resonant frequencies are increased which indicates that the capacitance is decreased which is in agreement with the principle discussed in Sect. II.

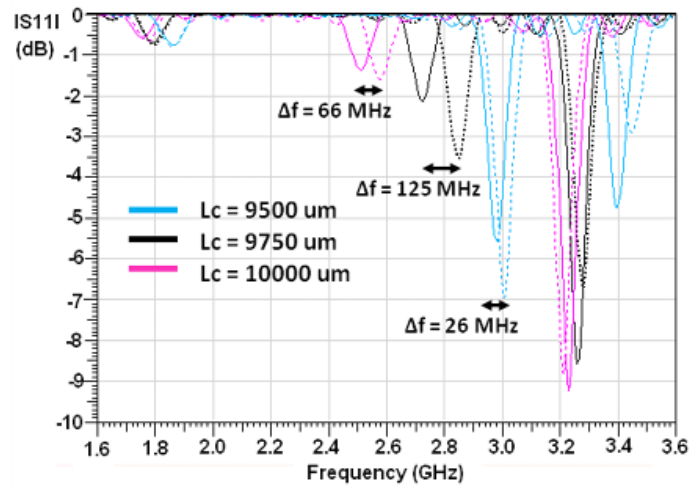

Fig. 5. Simulations of different Lc values.

\section{MEASUREMENT RESUltS AND REMOTE SENSING EXAMPLE}

\section{A. Measurement Results}

The first portion of the circuit was fabricated on Kapton 100um thick without the cantilever (Fig. 6a). The cantilever is fabricated on a separate substrate of Kapton 50um thick (Fig. $6 b)$. Then the cantilever is manually assembled onto the large circuit in LAAS. In the first portion of the circuit (Fig. 6a), the surface at the tip is cleaned and applied a small amount of transparent adhesive glue spread as thin as possible to create a 10-15um insulating layer, however not well controlled. As this glue layer is dry, the cantilever on Kapton 50um is soldered to the other end of the open loop (Fig. 8) with the copper layer faced down. Then to ensure the cantilever tip copper face is in contact with the glue layer in normal direction but freely to move in elongation direction, a buffer Kapton 127 um is placed on top of the cantilever and pressed down by Scotch tape. In this configuration, as the open loop is stretched, the cantilever can still move freely as the solder end of the cantilever pulls on it. This assembling configuration is illustrated in Fig. 7.

The measurement set up is also shown in Fig. 7 in which the strain is increased as the load is added. Measurements of the strain transducer are shown in Fig. 8. Notice that the load is added with irregular intervals due to limited availability of load. Because of such manual process, it is difficult to accurately control the initial gap capacitance of the circuit since the actually Lcap can be easily varied between 7501000 um. Therefore, it is difficult to tune the circuit's response to the highest sensitivity operation point (Fig. 5).
The resulting strain resulted from such setup can be estimated according to Eq. 3, where $\mathrm{P}$ is the load, E is the Young's modulus of Kapton, $\mathrm{t}$ is the thickness of Kapton, and $\mathrm{W}$ is the total width of the Kapton substrate which is $150 \mathrm{~mm}$.

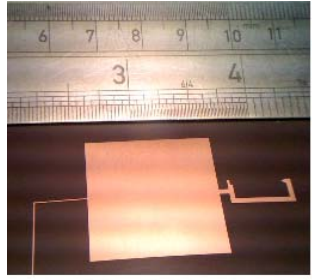

(a)

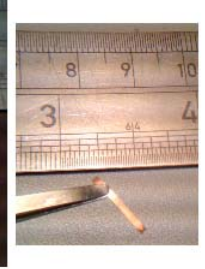

(b)
Fig. 6 Two different fabricated portions of the prototype.

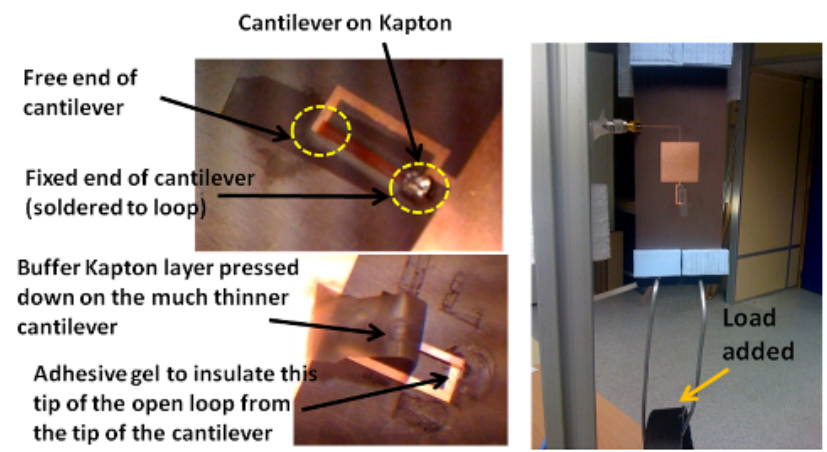

Fig 7. The assembled prototype and measurement setup.

$$
\varepsilon_{\mathrm{L}}=\frac{\mathrm{P}}{\mathrm{E} \cdot \mathrm{t} \cdot \mathrm{W}} \cdot 100 \%
$$

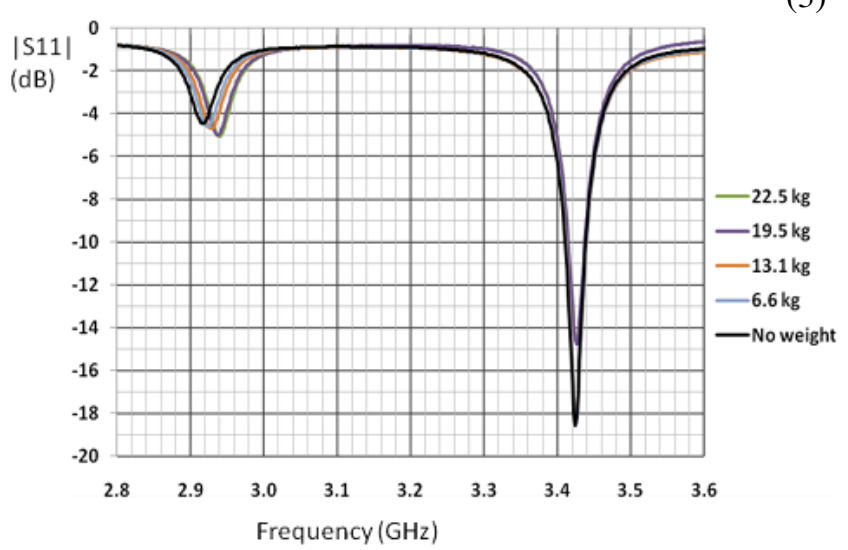

Fig. 8. Measurements of $|\mathrm{S} 11|$ of the fabricated prototype subjected to different weights attached.

The two resonant frequencies appear in Fig. 8 are $2.9 \mathrm{GHz}$ and $3.4 \mathrm{GHz}$ under no load, which is in excellent agreement with the simulation results as compared to the blue curve in Fig. 5 which is corresponding to the response of $\mathrm{Lc}=$ 9500um. In Fig. 11, a shift of $24 \mathrm{MHz}$ is recorded, from $2.914 \mathrm{GHz}$ with no load to $2.938 \mathrm{GHz}$ with $22.5 \mathrm{~kg}$ of load, corresponding to $0.645 \%$ strain, i.e. $1.26 \%$ frequency shift per $1 \%$ strain. The plot of percentage of frequency shift versus percentage of applied strain is shown in Fig. 9 indicating an excellent linear response for this strain transducer prototype within this range of strain. Although the prototype is not operating at the most sensitive frequency 
point as indicated by the black curve in Fig. 5, these measurements have successfully validated the novel design and the new principle introduced in this work.

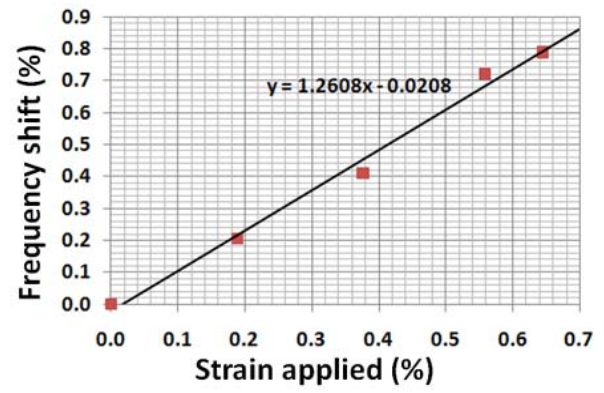

Fig. 5. Measurements of the proof-of-concept design.

\section{B. An Implementation Example for Remote Sensing based} on the new RF Strain Transducer

The RF strain transducer can be easily implemented in a completely passive network of sensing nodes to be interrogated remotely. The communication is based on the variation of the radar cross section (RCS) level of loaded multi-band scatterers read by a frequency-modulatedcontinuous-wave (FMCW) radar. The scatterers here are the patches loaded with the open loop rings, i.e. the strain transducers. In a sensing system, sensor nodes should not only provide information about physical conditions of the surrounding environment, but also provide their identification in the network of multiple sensor nodes. Therefore, based on the technique recently reported and illustrated in [6-7], the same concept is proposed for the implementation of the strain sensor to allow for an efficient communication between and discrimination within a network of many passive sensor nodes and a central monitoring station. The communication network is illustrated in Fig. 6.

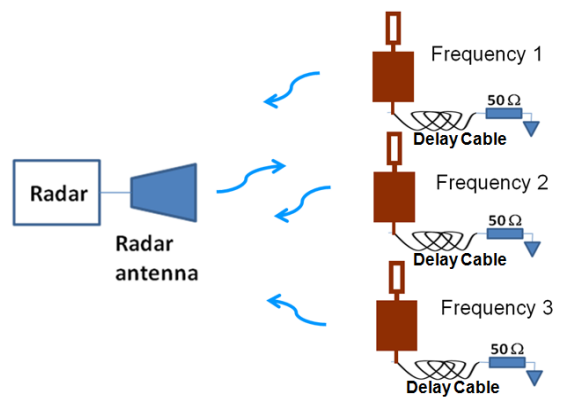

Fig. 6. RCS measurement system for remote sensing and identification.

The radar "reader" loaded horn antenna is usually located several meters away from the terminal of radar system. Delay cables are connected to different feeding points on the patches to terminate them with either match load, open or short. In this technique of remote identification and data acquisition, the radar emits RF signals, which would travel through the load system then reflected at the terminal and re-emitted from the sensors with some delays due to the RF cable. The radar ("reader") receives a modified response from the sensors while signals scattered from the rest of the environment remain unmodified. Based on the total travel distance of the signals, each object gives a different beat frequency with a different RCS level.

\section{CONCLUSIONS}

Strain passive and remote sensing for industrial structures as well as medical applications is highly desirable but was not quite practical. We have introduced a new design with novel principles for a highly sensitive wireless and passive RF strain sensor that is highly sensitive so it would allow such passive sensing system to be practically and efficiently realized. The design is illustrated and validated through simulations and measurements yielding a sensitivity of more than 4 times higher than the existing sensors in the same class. However, in experiments, the currently available prototype achieved a sensitivity of $1.26 \%$ frequency shift per $1 \%$ strain at $2.7-3$ $\mathrm{GHz}$ due to limited fabrication capability and rapid assembling. The sensor can also be easily scaled to operate at millimeter-wave frequencies for higher resolution of strain sensing, or adapted to biomedical applications at lower frequencies. With the well advanced MEMS technology and the simplicity of the design, low cost manufacturing approach of this design can be obtained. The new idea proposed in this work also enables a new dimension of engineered sensitivity in this type of strain sensing which previously was limited to the linear scale of the changes in the dimensions of the structures. The sensitivity of the proposed design is independent from the material of the applied surface due to the use of the ground plane. An example for a specific passive implementation of the sensor is illustrated in a radar system based on the monitoring of its RCS level change as an indicator of strain.

\section{ACKNOWLEDGMENT}

The authors would like to thank Tonio Idda for the generous help in measurements, Xavier Dollat and Sofiene Bouaziz for additional assistance in fabrication, as well as the support of LAAS-CNRS where this work was performed and the support of Georgia Tech.

\section{REFERENCES}

[1] U. Tara, H. Huang, R. Carter, et. al., "Exploiting a patch antenna for strain measurements," Mea. Sci. Tech., vol. 20(1), 2009.

[2] A. Daliri, A. Galehdar, S. John, et. al, "Circular Microstrip Patch Antenna Strain Sensor for Wireless Structural Health Monitoring," Proc. World Cong. Eng., vol. 2, London, UK, 2010.

[3] R. Melik, E. Unal, N. K. Perkgoz, et. al, "Metamaterial-based wireless strain sensors," Appl. Phys. Lett., vol. 95(1), 2009.

[4] D. Farhey, "Integrated virtual instrumentation and wireless monitoring for infrastructure diagnostics,"Str. Health Mon.,vol.5(1), pp.29-43, 2006.

[5] R. Chacon, F. Guzman, E. Mirambell, et. al, "Wireless sensor networks for strain monitoring during steel bridges launching," Str. Health Monitoring, vol. 8(3), pp. 195-205, 2009.

[6] M. M. Jatlaoui, F. Chebila, P. Pons, H. Aubert, "Wireless Interrogation Techniques for a Passive Pressure Micro-sensor using an EM Transducer," European Microwave Week, Nuova Fiera di Roma, Rome, Italy, 28 september - 2 October 2009.

[7] T. Thai, F. Chebila, M. Jatlaoui, P. Pons, H. Aubert, G. DeJean, M. Tentzeris, R. Plana, "Design and development of a millimetre-wave novel passive ultrasensitive temperature transducer for remote sensing and identification," Microwave Conference (EuMC), 2010 European, pp.45-48,Sept.2010. 